\title{
NONOSCILLATION THEOREMS FOR A SECOND ORDER SUBLINEAR ORDINARY DIFFERENTIAL EQUATION
}

\author{
MAN K.AM KWONG AND J. S. W. WONG
}

\begin{abstract}
A number of known and a conjectured nonoscillation criteria for sublinear Emden-Fowler equations are shown to be equivalent. One of these criteria is then extended to cover cases in which a growth condition on the coefficient of the equation is not satisfied.
\end{abstract}

1. We consider the second order Emden-Fowler equation

$$
y^{\prime \prime}(t)+a(t)|y(t)|^{\gamma} \operatorname{sgn} y(t)=0, \quad 0<\gamma<1,
$$

on $[0, \infty)$, where $a(t)$ is a positive continuous function. It is known that every solution is continuable throughout $[0, \infty)$ and under the additional assumption that $a(t)$ is locally of bounded variation on $[0, \infty)$, then (1) has a unique solution satisfying given initial conditions. Consequently, under these assumptions, which we make throughout, the set of zeros of any one particular solution of $(1)$ on $[0, \infty)$ is either finite or infinite extending to infinity with no accumulation points. Equation (1) is said to be nonoscillatory if every solution has only a finite number of zeros. For details, we refer to $[1,2,5,9]$.

Since $a(t)$ is assumed to be locally of bounded variation it admits a Jordan decomposition $a(t)=a_{+}(t)-a_{-}(t)$, where $a_{+}$and $a_{-}$are continuous nondecreasing functions. A number of nonoscillation results for the nonlinear equation (1), $\gamma \neq 1$, require some sort of restriction on the growth of the function $a(t)$, typically that $a(t)$ be nonincreasing. One such result contains the condition

$$
\int_{0}^{\infty} \frac{d a_{+}(t)}{a(t)}<\infty,
$$

due to Gollwitzer [5]. Under this condition, he proved the following three nonoscillation criteria for (1):

$$
\begin{gathered}
\int^{\infty} s a(s) d s<\infty \\
\lim _{t \rightarrow \infty} a^{(\gamma-1) / 2}(t) \int_{t}^{\infty} s^{\gamma} a(s) d s=0 \\
\lim _{t \rightarrow \infty} a^{(\gamma-1) / 2(\gamma+1)}(t) \int_{t}^{\infty} a^{1 / \gamma+1}(s) d s=0 .
\end{gathered}
$$

Received by the editors April !, 1982.

AMS ( MOS) subject classifications (1970). Primary 34C10, 34C15.

Key words and phrases. Second order, nonlinear, differential equations, oscillation.

(C) 1983 American Mathematical Society $0002-9939 / 82 / 0000-0867 / \$ 02.50$ 
Criterion (A) extends a result of Heidel [6] where the stronger assumption that $a(t)$ be nonincreasing is required. Recently, the second author proved the following nonoscillation criterion for equation (1) under the same assumption (2) (see [9]):

$$
\lim _{t \rightarrow \infty} t^{2} a(t)=0
$$

and showed that, under (2), condition (D) follows from condition (A). Earlier works of Coffman and Wong $[3,4]$ had introduced the concept of a "duality principle" between sublinear $(0<\gamma<1)$ and superlinear $(\gamma>1)$ equations, and as a result of this principle it was conjectured in [9] that condition (2) together with

$$
\lim _{t \rightarrow \infty} t \int_{t}^{\infty} a(s) d s=0
$$

also imply nonoscillation of (1). An application of L'Hôpital's rule shows that (D) implies $(E)$. However, the relationship of $(B)(C)$ to the others remains unclear, as does the sufficiency of (E) (with (2)) for nonoscillation. The purpose of this note is to prove the following result.

THEOREM 1. Under the growth assumption (2) (or more generally (3) below) on a $(t)$, the following chain of implications holds:

$$
(A) \Rightarrow(B) \Leftrightarrow(C) \Leftrightarrow(D) \Leftrightarrow(E) \text {. }
$$

The equivalence amongst (B), (C), (D) and (E) establishes (E) as a nonoscillation criterion for equation (1), proving the conjecture made in [9]. What is surprising though is that the seemingly very strong but simple condition (D) is just as good as (B), (C) or (E), so that when it comes to practical applications (D) is the only one needed. The chain of implications (**) also completes the comparison of nonoscillation between sublinear and superlinear equations under the general guidance of the "duality principle". Extensions to more general second order equations may be made much along the same lines as in our earlier work [4]. In \$5 we extend criterion (B) to equations for which (2) does not hold.

The proof of Theorem 1 is presented in the following way: in $\$ 2$ we prove that (D) $\Leftrightarrow(E)$; in $\$ 3$ we show that $(B) \Leftrightarrow(E)$ and finally in $\$ 4$ we show that $(C) \Rightarrow(D)$ and $(E) \Rightarrow(C)$. That $(A) \Rightarrow(D)$ under (2) has been established in [9]. The example $a(t)=\left[t^{2} \ln t\right]^{-1}$ shows that the converse is false.

Let us now note a possible reduction before we prove Theorem 1 .

LEMMA. If $a(t)$ is a positive continuous function of bounded variation on $[0, \infty)$ then it admits the decomposition

$$
a(t)=a_{1}(t) b(t), \quad t \geqslant 0,
$$

where $a_{1}(t)$ is a positive nonincreasing function and $b(t)$ is a positive nondecreasing function. If furthermore $a(t)$ satisfies (2), then $0<b_{1}<b(t) \leqslant b_{2}<\infty$ for all $t$ with fixed positive constants $b_{1}$ and $b_{2}$. 
Proof. This follows from the identity

$$
a(t)=a(0) \exp \left(-\int_{0}^{t} \frac{d a_{-}(s)}{a(s)}\right) \exp \left(\int_{0}^{t} \frac{d a_{+}(s)}{a(s)}\right) .
$$

Here, $b(t)=\exp \left(\int_{0}^{t} d a_{+} / a\right)$ tends to a finite limit as $t \rightarrow \infty$, if (2) holds.

Now suppose (2) holds. Using the fact that $b(t)$ is bounded above and below by positive constants, we see that $a(t)$ satisfies any of the conditions (B)-(E) if and only if $a_{1}(t)$ satisfies the same condition. Hence in the proof of Theorem 1 we can assume without loss of generality that $a(t)$ itself is nonincreasing.

2. Proof of $(D) \Leftrightarrow(E)$. The implication $(D) \Rightarrow(E)$ follows trivially from an application of L'Hôpital's rule.

Now suppose (E) holds but (D) does not hold. Then there exists a sequence $\left\{t_{n}\right\}$ tending to $\infty$ such that

$$
t_{n}^{2} a\left(t_{n}\right) \geqslant \delta>0
$$

for some constant $\delta$. By (E), if $t_{n}$ is large enough, then

$$
\frac{t_{n}}{2} \int_{t_{n} / 2}^{\infty} a(s) d s<\frac{\delta}{4} .
$$

On the other hand, since (4) holds and $a(t)$ is nonincreasing

$$
a(t) \geqslant \delta t_{n}^{-2}, \quad t \in\left[t_{n} / 2, t_{n}\right] .
$$

Integrating this inequality over $\left[t_{n} / 2, t_{n}\right]$ yields an inequality that contradicts (5).

3. Proof of $(B) \Leftrightarrow(E)$. We first prove that $(B) \Rightarrow(E)$. Given any $\varepsilon>0$, there exists a $t_{0}$ such that

$$
\left(\int_{t}^{\infty} s^{\gamma} a(s) d s\right)^{\alpha} \leqslant \varepsilon^{\alpha} a(t), \quad t \geqslant t_{0}
$$

where $\alpha=2 /(1-\gamma)$ is a positive constant. Letting $u(t)=\int_{t}^{\infty} s^{\gamma} a(s) d s$, we obtain from (6) $t^{\gamma} \leqslant-\varepsilon^{\alpha} u^{\prime}(t) / u^{\alpha}(t)$. Integrating this inequality we have

$$
u(t) \leqslant\left[\frac{(\alpha-1) t^{1+\gamma}}{(1+\gamma) \varepsilon^{\alpha}}+\frac{1}{u^{\alpha-1}\left(t_{0}\right)}\right]^{(\gamma-1) /(\gamma+1)}
$$

hence,

$$
\int_{t}^{\infty} s^{\gamma} a(s) d s=u(t)=O\left(\varepsilon^{2 /(\gamma+1)} t^{\gamma-1}\right),
$$

as $t \rightarrow \infty$. A simple integration by parts gives

$$
\begin{aligned}
\int_{t}^{T} a(s) d s & =\int_{t}^{T} s^{\gamma} a(s) \frac{1}{s^{\gamma}} d s \\
& =\frac{1}{t^{\gamma}} \int_{t}^{T} s^{\gamma} a(s) d s-\gamma \int_{t}^{T} \frac{1}{s^{1+\gamma}} \int_{s}^{T} \tau^{\gamma} a(\tau) d \tau d s .
\end{aligned}
$$


The fact that $\int_{t}^{T} s^{\gamma} a(s) d s \leqslant \int_{t}^{\infty} s^{\gamma} a(s) d s<\infty$ together with (9) show that $\int_{t}^{\infty} a(s) d s$ converges. Using (8) and letting $T \rightarrow \infty$ in (9), we obtain

$$
\int_{t}^{\infty} a(s) d s=O\left(\varepsilon^{2 /(1+\gamma)} t^{-1}\right) .
$$

Since $\varepsilon>0$ is arbitrary, (10) establishes the validity of $(E)$. Note that $(B) \Rightarrow(E)$ is established without recourse to the fact that $a(t)$ is nonincreasing, or (2) and (3).

Now suppose that (E) holds. Then for any $\varepsilon>0$, there exists $t_{0}$ such that $\int_{t}^{\infty} a(s) d s \leqslant \varepsilon / t$ for all $t \geqslant t_{0}$. An integration by parts yields the following identity:

$$
\int_{t}^{T} s^{\gamma} a(s) d s=t^{\gamma} \int_{t}^{T} a(s) d s+\gamma \int_{t}^{T} s^{\gamma-1} \int_{s}^{T} a(\tau) d \tau d s .
$$

The fact that $\int_{t}^{T} a(s) d s \leqslant \int_{t}^{\infty} a(s) d s=o\left(t^{-1}\right)$, as $t \rightarrow \infty$ implies that the right-hand side of (11), and hence also the left-hand side, converges as $T \rightarrow \infty$. Thus, $\int_{t}^{\infty} s^{\gamma} a(s) d s<\infty$. Furthermore, for $t \geqslant t_{0}$,

$$
\int_{t}^{\infty} s^{\gamma} a(s) d s \leqslant \varepsilon t^{\gamma-1} /(1-\gamma)=\left(\varepsilon_{1} / t\right)^{1-\gamma}
$$

where $\varepsilon_{1}^{1-\gamma}=\varepsilon /(1-\gamma)$. For any $t \geqslant t_{0}$, we consider the two mutually exclusive cases: (i) $a(t) \geqslant \varepsilon_{1} / t^{2}$ and (ii) $a(t)<\varepsilon_{1} / t^{2}$. In case (i), estimate (12) gives

$$
a^{(\gamma-1) / 2}(t) \int_{t}^{\infty} s^{\gamma} a(s) d s \leqslant\left(\frac{\varepsilon_{1}}{t^{2}}\right)^{(\gamma-1) / 2}\left(\frac{\varepsilon_{1}}{t}\right)^{1-\gamma}=\varepsilon_{1}^{(1-\gamma) / 2} .
$$

In case (ii), we set $b=\left(\varepsilon_{1} / a(t)\right)^{1 / 2}>t$ and observe that since $a(t)$ is nonincreasing it follows from (12) that

$$
\begin{aligned}
a^{(\gamma-1) / 2}(t) \int_{t}^{\infty} s^{\gamma} a(s) d s & =a^{(\gamma-1) / 2}(t)\left[\left(\int_{t}^{b}+\int_{b}^{\infty}\right) s^{\gamma} a(s) d s\right] \\
& \leqslant a^{(\gamma-1) / 2}(t)\left[\int_{0}^{b} s^{\gamma} a(t) d s+\left(\frac{\varepsilon_{1}}{b}\right)^{1-\gamma}\right] \\
& =\frac{\varepsilon_{1}^{(\gamma+1) / 2}}{\gamma+1}+\varepsilon_{1}^{(1-\gamma) / 2}=O\left(\varepsilon^{1 / 2}\right) .
\end{aligned}
$$

Since $\varepsilon>0$ is arbitrary, this established (B).

4. Proof of $(E) \Rightarrow(C)$ and $(C) \Rightarrow(D)$. To show $(E) \Rightarrow(C)$, we consider the identity, for $0<\gamma<\mu<1$,

$$
\int_{t}^{T} s^{\mu} a(s) d s=t^{\mu} \int_{t}^{T} a(s) d s+\mu \int_{t}^{T} s^{\mu-1} \int_{s}^{T} a(\tau) d \tau d s .
$$

Since $\int_{t}^{\infty} a=o(1 / t)$, we substitute it into (13) and find

$$
\int_{t}^{\infty} s^{\mu} a(s) d s=o\left(t^{\mu-1}\right) \text {. }
$$

Now we apply Schwarz's inequality and obtain

$$
\int_{t}^{\infty} a^{1 /(\gamma+1)}(s) d s \leqslant\left(\int_{t}^{\infty} s^{\mu} a(s) d s\right)^{1 /(\gamma+1)}\left(\int_{t}^{\infty} s^{-\mu / \gamma} d s\right)^{\gamma /(\gamma+1)} .
$$


(Here $p=\gamma+1>1, q=(\gamma+1) / \gamma$.) Substituting (14) into the right-hand side of (15), we obtain

$$
\int_{t}^{\infty} a^{1 /(\gamma+1)}(s) d s \leqslant o\left(t^{(\mu-1) /(\gamma+1)}\right)\left[\left(\frac{\gamma}{\mu-\gamma}\right) t^{(\gamma-\mu) / \gamma}\right]^{\gamma /(\gamma+1)}=o\left(t^{(\gamma-1) /(\gamma+1)}\right) .
$$

The remaining portion of the proof is patterned after that of $(E) \Rightarrow(B)$, which we repeat as follows. For any $\varepsilon_{2}>0$, we may fix $t_{0}$ from (16) so that for all $t \geqslant t_{0}$, we have

$$
\int_{t}^{\infty} a^{1 /(\gamma+1)}(s) d s \leqslant \varepsilon_{2}^{1 /(\gamma+1)} t^{(\gamma-1) /(\gamma+1)} .
$$

Now for any given $t \geqslant t_{0}$, we again consider two mutually exclusive cases: (i) $a(t) \geqslant \varepsilon_{2} / t^{2}$ and (ii) $a(t)<\varepsilon_{2} / t^{2}$. In the first instance, we obtain from (17) immediately the following estimate:

$$
[a(t)]^{(\gamma-1) / 2(\gamma+1)} \int_{t}^{\infty} a^{1 /(\gamma+1)}(s) d s \leqslant \varepsilon_{2}^{1 / 2} \quad(0<\gamma<1) .
$$

Otherwise, we have $a(t)<\varepsilon_{2} / t^{2}$. Set $b=\sqrt{\varepsilon_{2} / a(t)}>t$. Since $a(t)$ is nonincreasing, we first note

$$
\begin{aligned}
{[a(t)]^{(\gamma-1) / 2(\gamma+1)} \int_{t}^{\infty} a^{1 /(\gamma+1)}(s) d s } & \leqslant a^{1 / 2}(t)(b-t) \\
& \leqslant a^{1 / 2}(t) b=\varepsilon_{2}^{1 / 2}
\end{aligned}
$$

On the other hand, using estimate (17), we find that

$$
\begin{aligned}
{[a(t)]^{(\gamma-1) / 2(\gamma+1)} \int_{b}^{\infty} a^{1 /(\gamma+1)}(s) d s } & \leqslant[a(t)]^{(\gamma-1) / 2(\gamma+1)} \varepsilon_{2}^{1 /(\gamma+1)} b^{(\gamma-1) /(\gamma+1)} \\
& =\varepsilon_{2}^{1 /(\gamma+1)} \varepsilon_{2}^{(\gamma-1) / 2(\gamma+1)}=\varepsilon_{2}^{1 / 2}
\end{aligned}
$$

Combining (19) and (20), we have

$$
[a(t)]^{(\gamma-1) / 2(\gamma+1)} \int_{t}^{\infty} a^{1 /(\gamma+1)}(s) d s \leqslant 2 \sqrt{\varepsilon_{2}} .
$$

Since $\varepsilon_{2}>0$ is arbitrary, (18) and (21) establish the desired assertion (C).

We now show $(C) \Rightarrow(D)$.

Let $\alpha=2 /(1-\gamma)>1$ and $1 / \beta=\alpha-1=(\gamma+1) /(1-\gamma)>1$. Choose $\varepsilon^{\alpha}<$ $(\alpha-1) 4^{-\alpha}$. By (C) there exists $t_{0}$ such that for all $t \geqslant t_{0}$..

$$
[a(t)]^{(\gamma-1) / 2(\gamma+1)} \int_{t}^{\infty} a^{1 /(\gamma+1)}(s) d s<\varepsilon .
$$

Denote $f(t)=a^{\beta / 2}(t)$, then $f^{\alpha}(t)=a^{1 /(\gamma+1)}(t)$. Suppose that (D) fails. Then there exist constants $c$ and $\delta>0$ such that $c \geqslant 2 t_{0}$ and $a(c) \geqslant \delta / c^{2}$, or

$$
f^{\alpha}(c) \geqslant \bar{\delta} c^{-2 /(\gamma+1)},
$$

where

$$
\bar{\delta}=\delta^{1 /(\gamma+1)}>0,
$$


from which it follows with $d=\frac{3}{4} c$ that

$$
\int_{d}^{c} f^{\alpha}(s) d s \geqslant f^{\alpha}(c) \frac{c}{4} \geqslant \frac{1}{4} \bar{\delta} c^{-\beta} .
$$

Notice here although the choice of $c$ depends on $\varepsilon$, that of $\delta$ or $\bar{\delta}$ does not. We now rewrite (22) in terms of $f(t)$ as

$$
f(t)>\frac{1}{\varepsilon} \int_{t}^{\infty} f^{\alpha}(s) d s>\frac{1}{\varepsilon}\left[\int_{t}^{d} f^{\alpha}(s) d s+\int_{d}^{c} f^{\alpha}(s) d s\right]
$$

for $t_{0} \leqslant c / 2 \leqslant t \leqslant \frac{3}{4} c$. Using (25) in the above estimate, we find

$$
f(t)>\frac{1}{4 \varepsilon} \bar{\delta} c^{-\beta}+\frac{1}{\varepsilon} \int_{t}^{d} f^{\alpha}(s) d s .
$$

Now define $g(x)=f(t)=f(d-x)$, then for $0 \leqslant x \leqslant c / 4,(26)$ becomes

$$
g(x)>\frac{1}{4 \varepsilon} \bar{\delta} c^{-\beta}+\frac{1}{\varepsilon} \int_{0}^{x} g^{\alpha}(u) d u .
$$

Compare $g(x)$ in the above with its minorant function $G(x)$ defined by

$$
G(x)=\frac{1}{4 \varepsilon} \bar{\delta} c^{-\beta}+\frac{1}{\varepsilon} \int_{0}^{x} G^{\alpha}(u) d u .
$$

An application of standard results for integral inequalities (see e.g. Walter [7]) to (27) and (28), gives $g(x) \geqslant G(x)$ for $0 \leqslant x \leqslant c / 4$. The function $G(x)$ can be found by explicitly solving (28); thus

$$
G(x)=\left[G^{1-\alpha}(0)+(1-\alpha) \frac{x}{\varepsilon}\right]^{-\beta}
$$

where $G^{1-\alpha}(0)=\left(\bar{\delta} c^{-\beta} / 4 \varepsilon\right)^{-1 / \beta}=(4 \varepsilon / \bar{\delta})^{1 / \beta} c$. From (29), one finds

$$
G(x) \rightarrow+\infty \quad \text { as } x \rightarrow \frac{\varepsilon}{(\alpha-1)}\left(\frac{4 \varepsilon}{\bar{\delta}}\right)^{1 / \beta} c \text {. }
$$

So unless

$$
\frac{\varepsilon}{(\alpha-1)}\left(\frac{4 \varepsilon}{\bar{\delta}}\right)^{1 / \beta}>\frac{1}{4},
$$

$G(x)$ would blow up on the finite interval [0,c/4], and so would $g(x)$. This would contradict the fact that $f(t)$ is defined on $\left[\frac{1}{2} c, \frac{3}{4} c\right]$. Recall that $\bar{\delta}$ is determined only from the fact that (D) fails and is independent of the choice of $\varepsilon$. It follows that (30) must hold for all $\varepsilon>0$, an obvious impossibility. This completes the proof of $(C) \Rightarrow$ (D) and also that of Theorem 1.

5. Extension of criterion (B). In case condition (2) does not hold, we have the following extension of (B).

THEOREM 2. Let $a_{1}(t)=\exp \left(-\int_{0}^{t} d a_{-}(s) / a(s)\right)$. If

$$
\lim _{t \rightarrow \infty} a_{1}^{(\gamma-1) / 2}(t) \int_{t}^{\infty} s^{\gamma} a(s) d s=0
$$

then the sublinear Emden-Fowler equation (1) is nonoscillatory. 
Proof. Differentiating the energy function

$$
\phi(t)=\gamma^{\prime 2}(t) / 2 a(t)+|y(t)|^{\gamma+1} /(\gamma+1)
$$

we find that

$$
d \phi(t)=-\frac{d a(t)}{a^{2}(t)} \frac{y^{\prime 2}(t)}{2} \geqslant-\frac{d a_{+}(t)}{a(t)} \phi(t) .
$$

It follows that

$$
\phi(t) \geqslant \phi(0) \exp \left(-\int_{0}^{t} \frac{d a_{+}(s)}{a(s)} d s\right)=\phi(0) / b(t)
$$

where $b(t)$ is as defined in the Lemma in $\$ 1$.

Now suppose that (1) is not nonoscillatory, i.e. there exists a solution $y$ with arbitrarily large zeros. Let $\tau$ be one of these zeros chosen so large that

$$
a_{1}^{(\gamma-1) / 2}(\tau) \int_{\tau}^{\infty} s^{\gamma} a(s) d s<[2 \phi(0)]^{(1-\gamma) / 2} .
$$

Without loss of generality we may assume that $y^{\prime}(\tau) \geqslant 0$ so that $y$ is positive in $[\tau, \bar{\tau}]$ where $\bar{\tau}$ is the next zero immediately after $\tau$. By (32),

$$
\frac{y^{\prime 2}(\tau)}{2 a(\tau)}=\phi(\tau) \geqslant \frac{\phi(0)}{b(\tau)} .
$$

It follows from this inequality and the Lemma that

$$
y^{\prime 2}(\tau) \geqslant 2 \phi(0) \frac{a(\tau)}{b(\tau)}=2 \phi(0) a_{1}(\tau) .
$$

Since $y$ is concave in $[\tau, \bar{\tau}], y^{\prime}(t) \leqslant y^{\prime}(\tau)$ for $t \in[\tau, \bar{\tau}]$. Thus

$$
y(t) \leqslant y^{\prime}(\tau)(t-\tau) \leqslant y^{\prime}(\tau) t, \quad t \in[\tau, \bar{\tau}] .
$$

Using (1) we see that $-y^{\prime \prime}(t) \leqslant\left[y^{\prime}(\tau)\right]^{\gamma} t^{\gamma} a(t)$. Integrating yields

$$
y^{\prime}(\tau)-y^{\prime}(t) \leqslant\left[y^{\prime}(\tau)\right]^{\gamma} \int_{\tau}^{t} s^{\gamma} a(s) d s .
$$

In particular when $t=\bar{\tau}$, we have

$$
y^{\prime}(\tau) \leqslant\left[y^{\prime}(\tau)\right]^{\gamma} \int_{\tau}^{\bar{\tau}} s^{\gamma} a(s) d s \leqslant\left[y^{\prime}(\tau)\right]^{\gamma} \int_{\tau}^{\infty} s^{\gamma} a(s) d s .
$$

Thus $\left[y^{\prime}(\tau)\right]^{1-\gamma} \leqslant \int_{\tau}^{\infty} s^{\gamma} a(s) d s$. Substituting (34) into this inequality we obtain an inequality that contradicts (33). This completes the proof.

\section{REFERENCES}

1. F. V. Atkinson, On second order nonlinear oscillations, Pacific J. Math. 5 (1955), 643-647.

2. C. V. Coffman and D. F. Ullrich, On the continuation of solutions of a certain nonlinear differential equation. Monatsh. Math. 71 (1967), 385-392.

3. C. V. Coffman and J. S. W. Wong, Oscillation and nonoscillation of solutions of generalized Emden-Fowler equations, Trans. Amer. Math. Soc. 167 (1972), 399-434.

4. Oscillation and nonoscillation theorems for second order ordinary differential equations, Funkcial. Ekvac. 15 (1972), 119-130. 
5. M. E. Gollwitzer, Nonoscillation theorem for a nonlinear differential equation, Proc. Amer. Math. Soc. 26 (1970), 78-84.

6. J. W. Heidel, $A$ nonoscillation theorem for a nonlinear second order differential equation, Proc. Amer. Math. Soc. 22 (1969), 485-488.

7. W. Walter, Differential and integral inequalities, Springer-Verlag, Berlin and New York, 1970.

8. J. S. W. Wong, On the generalized Emden-Fowler equation, SIAM Rev. 17 (1975), 339-360.

9. Remarks on nonoscillation theorems for a second order nonlinear differential equation, Proc. Amer. Math. Soc. 83 (1981), 541-546.

Department of Mathematical SCiences, Northern Illinois University, DeKalb, Illinois 60115 (Current address of M. K. Kwong)

Department of MAThematics, University of Hong Kong, Hong Kong

Current address: (J. S. W. Wong): China Dyeing Works, Ltd., 819 Swire House, Hong Kong 